\title{
The nuclear ring in the unbarred galaxy NGC 278: Result of a minor merger?
}

\author{
J. H. Knapen ${ }^{1}$, L. F. Whyte ${ }^{2}$, W. J. G. de Blok ${ }^{3}$, and J. M. van der Hulst ${ }^{4}$ \\ ${ }^{1}$ Centre for Astrophysics Research, University of Hertfordshire, Hatfield, Herts AL10 9AB, UK \\ e-mail: j . knapen@star.herts.ac.uk \\ 2 University of Nottingham, School of Physics and Astronomy, University Park, Nottingham, NG7 2RD, UK \\ 3 Department of Physics and Astronomy, Cardiff University, 5 The Parade, Cardiff CF24 3YB, UK \\ 4 Kapteyn Astronomical Institute, University of Groningen, Postbus 800, 9700 AV Groningen, The Netherlands
}

Received 17 September 2003 / Accepted 24 April 2004

\begin{abstract}
We present fully sampled high angular resolution two-dimensional kinematics in the $\mathrm{H} \alpha$ spectral line, optical and near-infrared imaging, as well as $21 \mathrm{~cm}$ atomic hydrogen data of the spiral galaxy NGC 278 . This is a small non-barred galaxy, which has a bright star forming inner region of about $2 \mathrm{kpc}$ in diameter, reminiscent of nuclear rings seen mainly in barred galaxies. The $\mathrm{H} \alpha$ kinematics show a disturbed velocity field, which may be partly the result of spiral density wave streaming motions. The $21 \mathrm{~cm}$ data trace the atomic hydrogen well outside the optical disk. The $\mathrm{H}_{\mathrm{I}}$ is not abundant but clearly shows disturbed morphology and kinematics. We postulate that the current structure of NGC 278 is a result of a recent interaction with a small gas-rich galaxy, which is now dispersed into the outer disk of NGC 278. Non-axisymmetries set up in the disk by this minor merger may well be the cause of the intense star formation in the inner region, which can be interpreted as a rare example of a nuclear ring in a non-barred galaxy. Rather than being induced by a bar, this nuclear ring would then be the direct result of an interaction event in the recent history of the galaxy.
\end{abstract}

Key words. galaxies: individual: NGC 278 - galaxies: ISM - galaxies: kinematics and dynamics - galaxies: spiral galaxies: structure

\section{Introduction}

Rings in disk galaxies are intimately linked to the internal dynamics and the evolution of their hosts. Rings are common, are more often than not sites of recent massive star formation (SF), and are linked observationally and phenomenologically to the presence of bars (see Buta \& Combes 1996 for a general review on galactic rings). Nuclear rings occur in about one fifth of spiral galaxies (Knapen 2004). They are found almost exclusively in barred galaxies (e.g., Buta \& Combes 1996; Knapen 2004), and can be directly linked to inner Lindblad resonances (ILRs), between the epicyclic oscillations of the stars and the rotation of the (bar) pattern (Knapen et al. 1995a; Heller \& Shlosman 1996; Shlosman 1999). Because of the much enhanced massive SF occurring in nuclear rings, they are prime tracers of SF processes in starburst regions, as well as of the dynamics of galaxies close to the nucleus. Although nuclear rings are intimately connected to bar dynamics, in rare cases a nuclear ring occurs in a galaxy where no bar can be identified. In this paper, we study one such case, that of NGC 278, in detail.

We describe high resolution $\mathrm{H} \alpha$ Fabry-Pérot (FP) data, in combination with optical and near-infrared (NIR) imaging and with $\mathrm{H}_{\mathrm{I}} 21 \mathrm{~cm}$ synthesis data, of the rather face-on
SAB(rs)b (de Vaucouleurs et al. 1991, hereafter RC3) spiral galaxy NGC 278 (UGC 528). NGC 278 has several knotty spiral arms, first described by Pease (1917). Pease also noticed the sudden drop in intensity at about 50 arcsec in diameter, which is just outside the region where the much enhanced SF is organised into a nuclear ring (e.g., Pogge 1989; Sect. 4.5) of about 40 arcsec, or some $2.2 \mathrm{kpc}$, in diameter. The galaxy is small $\left(D_{25}=2.1\right.$ arcmin, RC3; or a diameter of $7.2 \mathrm{kpc}$, Tully 1988) but has an $\mathrm{H}$ I disk which extends significantly further out, as we will show below. At a distance of 11.8 Mpc (Tully 1988), 1 arcsec corresponds to $57 \mathrm{pc}$.

NGC 278 is part of the local supercluster of galaxies centred around the Virgo cluster (Vallee 1993). Arp \& Sulentic (1985), in their analysis of groups of galaxies, listed NGC 278 as having a companion dwarf galaxy UGC 672. In their study of interacting galaxies, Bushouse et al. (1988), however, considered NGC 278 to be an isolated galaxy, which is in agreement with Karachentseva et al. (1996) who found UGC 672 to be an isolated dwarf galaxy. Although the difference in systemic velocity between the two galaxies is only $68 \mathrm{~km} \mathrm{~s}^{-1}$ (RC3), combining their positions on the sky with the scale as given in the previous paragraph, we find that NGC 278 and UGC 672 are some $600 \mathrm{kpc}$ distant, too much to consider them interacting, 
especially when considering the faint absolute magnitude of UGC 672 , of about -12.4 (estimated using parameters from the RC3, in comparison, $M_{B}=-18.8$ for NGC 278). We will come back to interactions later in this paper.

Pogge (1989) reported a round, 11 arcsec (450 pc using our distance assumption) diameter, nuclear ring "embedded in a bright, starbursting disk" from $\mathrm{H} \alpha$ imaging. Deconvolved $F 555 W$ (roughly $V$-band) images of NGC 278 taken with the Hubble Space Telescope (HST) WFPC revealed evidence for the existence of spiral structure to within 2-3 arcsec of the unresolved nucleus, and for a narrow dust lane approaching within 0.5 arcsec of the very centre (Phillips et al. 1996). Schmidt et al. (1990) used a combination of optical spectroscopy and stellar population synthesis modelling to study the central region of NGC 278, and suggest that the predominant HII regions are in late evolutionary stages. The high luminosity of the galaxy is almost certainly caused by intense SF, a burst of which is still operating (Schmidt et al. 1990). More recently, Garrido et al. (2003) published FP observations of, among other galaxies, NGC 278, obtained as part of their GHASP survey. Their data, at a spatial resolution of $2 \operatorname{arcsec}$ or almost twice as large as our data, show roughly the same morphological and velocity structure as presented in the current paper. Garrido et al. (2003) claim that NGC 278 shows a barred spiral structure, although it is not clear on the basis of which source they may have reached this conclusion, and also that the isovelocity contours confirm the presence of a bar. We will show below, on the basis not only of FP $\mathrm{H} \alpha$ data but also of a large quantity of optical and NIR imaging, as well as the WHISP H I data to which Garrido et al. briefly refer in their paper, that NGC 278 does not show any evidence for the presence of a bar, and that the behaviour of its gaseous velocity field, as well as the presence of the nuclear ring of SF, is a likely result of a past interaction.

After describing the FP and Hi data and our optical, NIR and HST imaging (Sect. 2), we detail the morphological and kinematic results in Sect. 3. We discuss the spiral structure, nuclear ring, and the overall morphology, as well as the plausible recent history of the galaxy, in Sect. 4, and summarise our main results in Sect. 5 .

\section{Observations and data reduction}

\subsection{Fabry-Pérot data}

The $\mathrm{H} \alpha$ FP maps were obtained on the night of 1998 September 2 with the TAURUS-II instrument, equipped with a TEK CCD camera, on the $4.2 \mathrm{~m}$ William Herschel Telescope (WHT) on La Palma. The data reduction procedures are summarised below, but are very similar to those used by Knapen et al. (2000) and Laine et al. (2001), to which we refer for further details. For the data set of NGC 278, the CCD was windowed to 400 pixels $\times 400$ pixels of $0.28 \operatorname{arcsec}_{\text {pixel }}{ }^{-1}$. The night was photometric with sub-arcsecond seeing. We used an appropriately redshifted narrow band $\mathrm{H} \alpha$ filter as an order-sorting filter $\left(\lambda_{\mathrm{c}}=6577 \AA, \Delta \lambda=15 \AA\right.$, using the galaxy's systemic velocity $v_{\text {sys }}=640 \mathrm{~km} \mathrm{~s}^{-1}$ ). Wavelength and phase calibration were performed using observations of a calibration lamp before and after each science exposure. The final calibrated data cube has 400 pixels $\times 400$ pixels $\times$ 55 "planes" in wavelength, the latter separated by $0.405 \AA$, or $18.5 \mathrm{~km} \mathrm{~s}^{-1}$. The spatial resolution of the resulting data set is $\sim 1.1$ arcsec. The data set was placed on an astrometrically correct grid by comparing the positions of point-like features in the $\mathrm{H} \alpha$ FP data with those in the broad-band images (see below), which in turn could be referenced to astrometry from a digitised sky survey image using fits to positions of foreground stars.

We determined which channels of the data set were free of $\mathrm{H} \alpha$ line emission after smoothing it to a resolution of $4 \operatorname{arcsec} \times$ 4 arcsec. Subsequently, we subtracted the continuum emission after fitting the continuum to the 15 line-free channels on either extreme of the data cube. The $\mathrm{H} \alpha$ emission in the slightly smoothed data set (to 2 arcsec spatial resolution), as a function of increasing wavelength, or velocity, is shown as a set of channel maps in Fig. 1. In order to increase the signal-to-noise ratio across the region of the galaxy where $\mathrm{H} \alpha$ emission is detected, we smoothed the original data with Gaussians to produce a number of similar data sets at spatial resolutions of 1.2, 1.5, 2, 3 and 4 arcsec.

Following the procedures described in detail by Knapen (1997) and Knapen et al. (2000), we produced moment maps of the data cubes at different spatial resolution. Moment zero ( $\mathrm{H} \alpha$ total intensity) and one (velocity field) maps at a spatial resolution of 1.2 arcsec are shown in Fig. 2.

\subsection{NIR imaging}

We obtained $J, H$ and $K^{\prime}$ images of NGC 278 on the night of 1995 November 5 using the $3.6 \mathrm{~m}$ Canada-France-Hawaii Telescope (CFHT) and the Montreal NIR camera (MONICA; Nadeau et al. 1994), equipped with a $256 \times 256$ pixel $\mathrm{HgCdTe}$ array detector with a projected pixel size of 0.248 arcsec. Full details of the image reduction can be found in Pérez-Ramírez et al. (2000). The resolution of the NIR images is slightly better than 1 arcsec. We show our NIR imaging results in Fig. 3 in the form of grey-scale and contour plots of the $J, H$ and $K^{\prime}$ images, and a $J-K^{\prime}$ colour index image. The NIR images have not been photometrically calibrated, but we will assume an average colour of $J-K=0.85$, as obtained from Frogel et al. (1978) using an absolute magnitude of the galaxy of $M_{B}=-18.8$ (derived from the RC3 and the assumed distance).

\subsection{Ground-based optical imaging}

Optical images in the $B$ and $R$ bands were obtained from the Isaac Newton Group's (ING) data archive. The images, of 300 s exposure time each, were obtained on 1995 December 23 with the prime focus camera on the Isaac Newton Telescope, and were flat-fielded by us using standard procedures. The TEK3 CCD gives a pixel scale of 0.59 arcsec pixel ${ }^{-1}$, and the seeing as measured on the images was 1.1 arcsec. Photometric calibration was obtained from exposures of the standard star field PG 0231+051. Grey-scale and contour representations of 


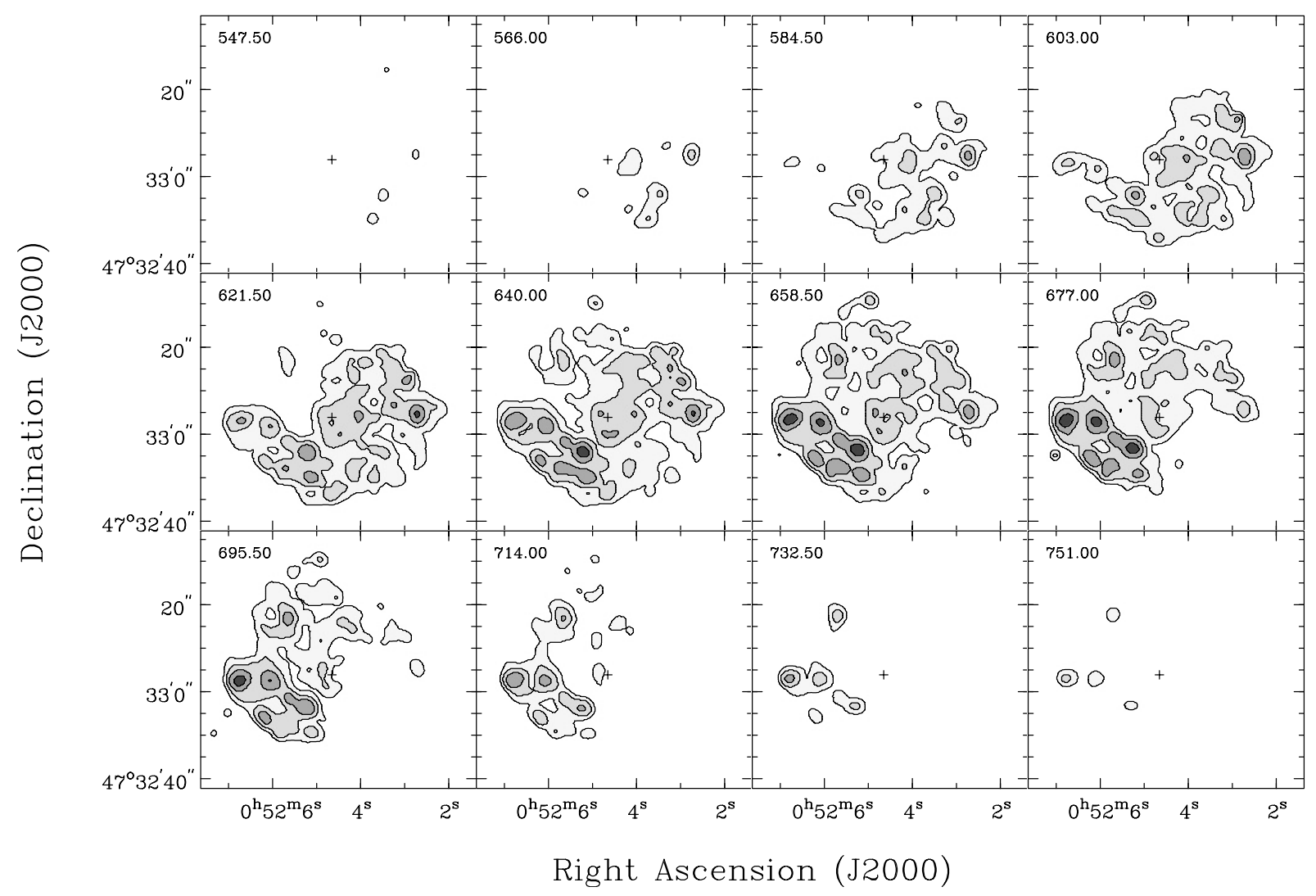

Fig. 1. Channel maps of the $\mathrm{H} \alpha$ emission from NGC 278 at 2 arcsec resolution. Velocity in $\mathrm{km} \mathrm{s}^{-1}$ of each channel is indicated in the upper left corner. Contour and grey-scale levels are 3 to $15 \sigma$ in steps of $3 \sigma$, with an additional contour at $-3 \sigma$. The kinematic centre of the galaxy is marked with a cross.

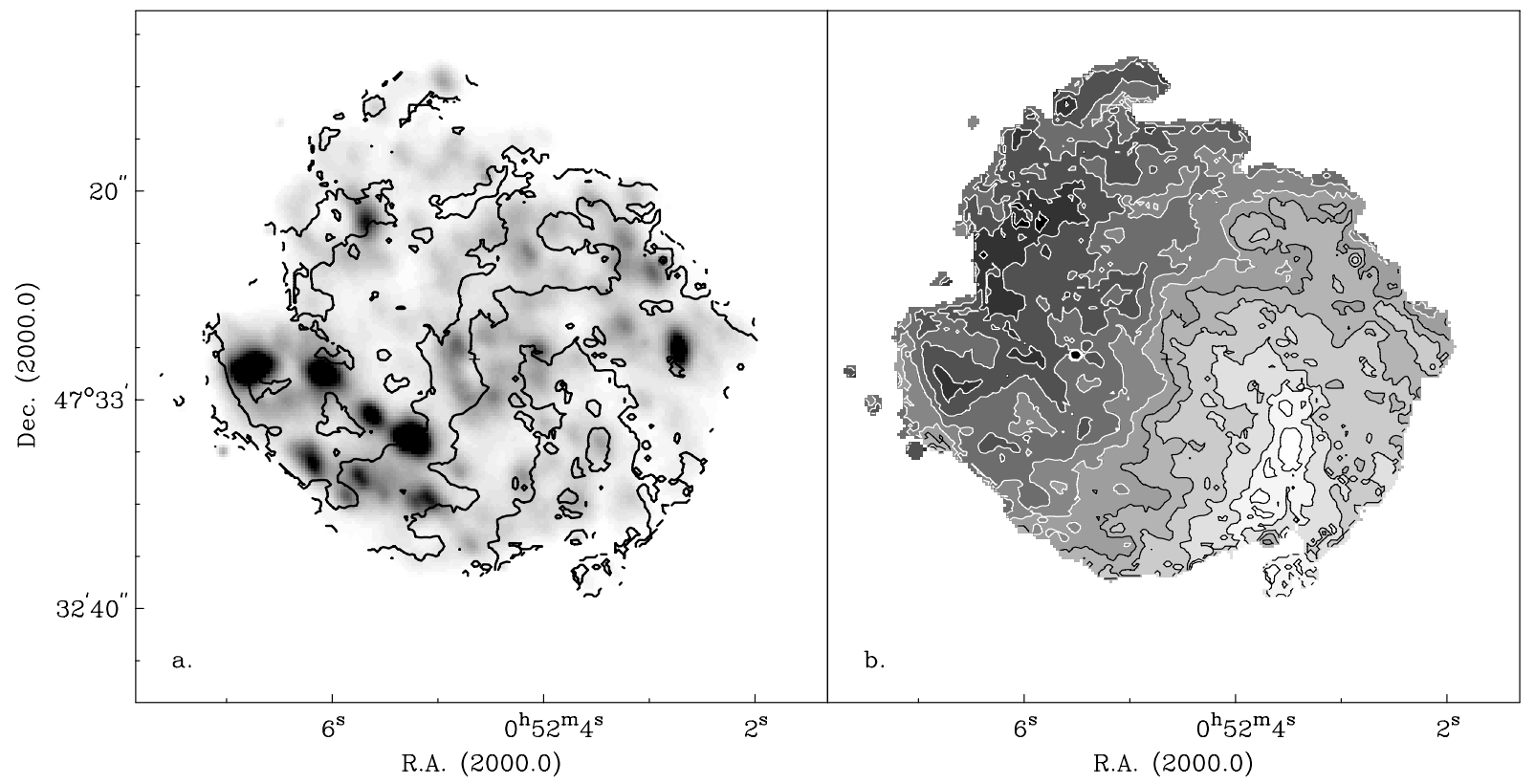

Fig. 2. Moment maps of the FP data set of NGC 278 at 1.2 arcsec resolution: $\mathrm{H} \alpha$ total intensity map (left) and velocity field (right). Grey-scale and contour levels in the right panel are from 600 to $690 \mathrm{~km} \mathrm{~s}^{-1}$ in steps of $10 \mathrm{~km} \mathrm{~s}^{-1}$, with the lower velocity contours in black, and the first white contour at $650 \mathrm{~km} \mathrm{~s}^{-1}$ (darker grey shades thus indicate higher velocities). In the left panel, only every second velocity contour is shown. The kinematic centre of the galaxy is marked with a cross.

the $B$ and $R$ images, and of a $B-R$ colour index image, are shown in Fig. 4.
These optical images of the galaxy are influenced by stray light, and CCD charge overflow, from the $8.8 \mathrm{~B}$-mag 


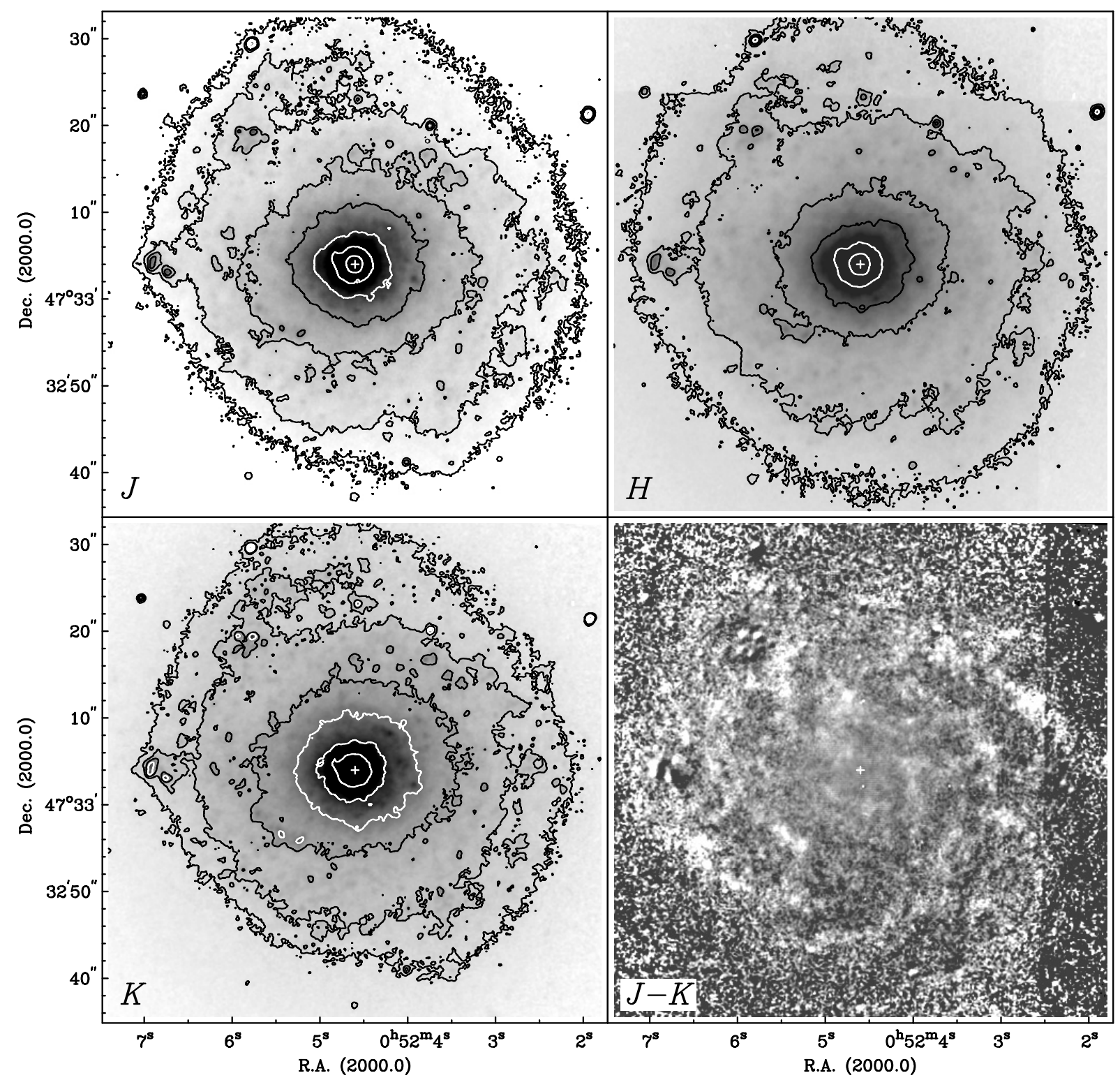

Fig. 3. NIR $J, H$, and $K^{\prime}$-band images, with contour levels set at steps of $0.5 \mathrm{mag}$, and (bottom right) grey-scale representation of the $J-K^{\prime}$ colour index image, with redder regions shown as darker shades.

A0 star HD 4950, located just 2.5 arcmin north of the centre of NGC 278.

\subsection{HST imaging}

From the $H S T$ archive, we obtained images taken through the $F 450 \mathrm{~W}, F 606 \mathrm{~W}$, and $F 814 \mathrm{~W}$ filters with the WFPC2 camera. The images were taken on 2001 August 20, and form part of a snapshot survey GO 9042, the PI of which is Dr. Stephen Smartt. Two images of the galaxy, of $160 \mathrm{~s}$ each, were taken through each filter. Whereas the INT images (Sect. 2.3) are deeper and show the faint outer structure in more detail, the $H S T$ images show the structure in the inner kpc region in much clearer detail thanks to the high spatial resolution. We present the HST images, combined into a real-colour view of the galaxy, in Fig. 5.

\subsection{HI data}

NGC 278 was observed as part of the WHISP project (van der Hulst et al. 2001; van der Hulst 2002). One of the standard products of the survey are data cubes at the full resolution of the Westerbork Synthesis Radio Telescope (WSRT) array ( $\sim 15 \operatorname{arcsec})$, at $30 \operatorname{arcsec}$, and at 60 arcsec. In addition, we created a low-resolution data cube with a beam size of 120 arcsec. We produced total surface density maps and velocity fields at each of the resolutions. We first isolated all signal over $2 \sigma$ in the 120 arcsec data, and, after removing spurious noise peaks, used this as a mask for the 60 arcsec data in order to create the 60 arcsec moment maps. We then isolated all signal brighter than $2 \sigma$ in the 60 arcsec masked map and used this as a mask for the 30 arcsec data, and similarly for the full resolution data. We show the Hi surface density maps at 60 and 120 arcsec 

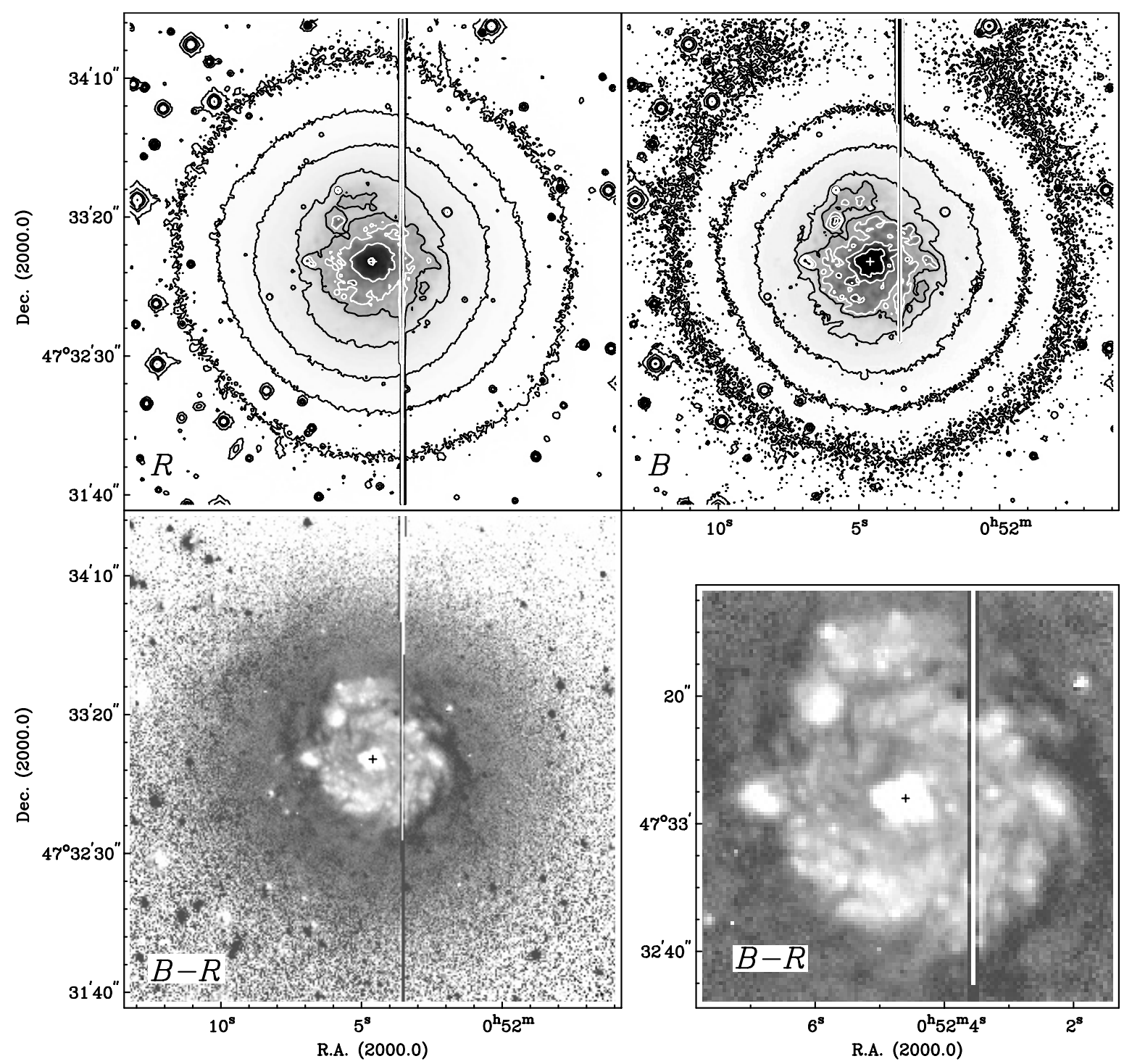

Fig. 4. Top panels: grey-scale and contour plots of the $R$ and $B$-band images, with in the $R$-band, black contours from 23.5 to 19.5 in steps of $1 \mathrm{mag} \operatorname{arcsec}^{-2}$ and white contours at 19.0, 18.5 and $18.0 \mathrm{mag} \mathrm{arcsec}{ }^{-2}$, and in the $B$-band black contours from 24.8 to 20.8 in steps of $1 \mathrm{mag} \operatorname{arcsec}^{-2}$ and white contours at 20.3, 19.8 and $19.3 \mathrm{mag} \operatorname{arcsec}^{-2}$. Lower panels: grey-scale plot of the $B-R$ colour index image (left), with a closeup of the central region (right). Darker shades are redder. Vertical lines are due to a bright star located about 2.5 arcmin to the North of the galaxy. A cross marks the centre of the galaxy.

resolution in Fig. 6, and the velocity field at 120 arcsec resolution in Fig. 7.

The data covers the entire primary beam of the WSRT array ( 30 arcmin), and spans a velocity range from $380 \mathrm{~km} \mathrm{~s}^{-1}$ to $902 \mathrm{~km} \mathrm{~s}^{-1}$ with a channel separation of $4.14 \mathrm{~km} \mathrm{~s}^{-1}$. The rms noise in the 60 arcsec and 120 arcsec data which we use in this paper is $5 \mathrm{mJy}_{\text {beam }}^{-1}$ and $7.6 \mathrm{mJy} \mathrm{beam}^{-1}$, respectively.

\section{Results}

\subsection{Morphology}

\subsubsection{Optical morphology of the circumnuclear region and inner disk}

NGC 278 is characterised by the strong dualistic behaviour of the SF in its disk, with strong SF in the inner $2 \mathrm{kpc}$ diameter region, and hardly any SF at all outside this sharply defined radius. This can be seen in the images (Figs. 3-5) and most clearly in the radial surface brightness and especially colour profiles (Fig. 8). The latter shows that within a radial range of some 5 arcsec, the colour becomes redder by at least $0.3 \mathrm{mag}$. The bluer colour within $r=26$ arcsec is caused by enhanced $\mathrm{SF}$, as evidenced by, e.g., the $\mathrm{H} \alpha$ morphology (Fig. 2).

The outer disk, at radii larger than 27 arcsec or $1.5 \mathrm{kpc}$, is almost completely featureless as best seen in optical broad bands (Fig. 4). A fit to the exponential disk in the radial range from 100 to 180 arcsec gives a scale length of $51 \operatorname{arcsec}$ in $B$ and $43 \operatorname{arcsec}$ in $R$. These fits are indicated as straight lines in the radial surface brightness profiles (Fig. 8). Our H $\alpha$ FP imaging shows a complete lack of massive SF activity outside the sharp dividing radius of 27 arcsec. The spiral structure, so prominent in the inner region, does continue into the outer 


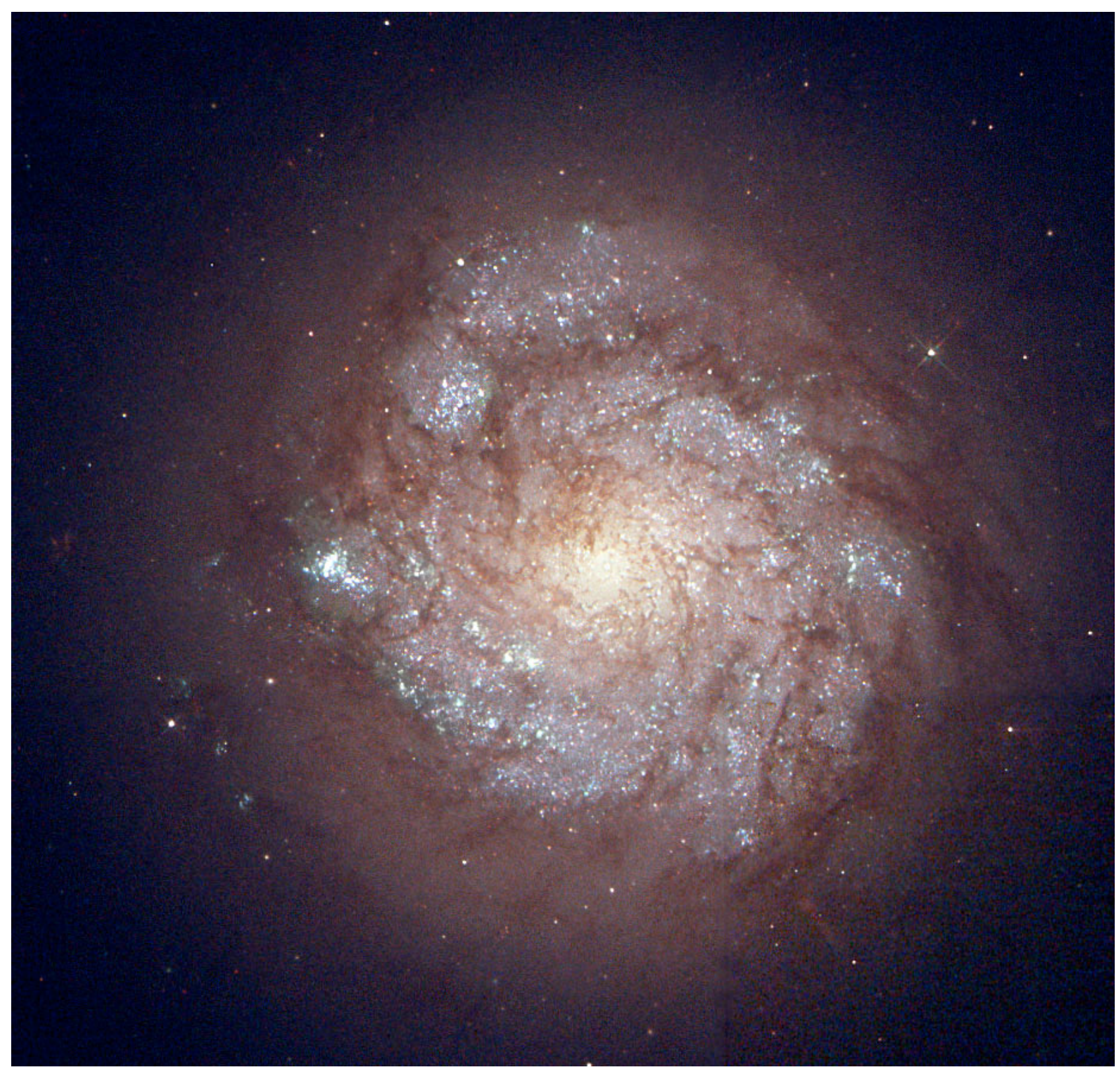

Fig. 5. Real-colour image made from the $F 450 \mathrm{~W}, F 606 \mathrm{~W}$, and $F 814 \mathrm{~W} H S T$ images. Area shown is about 100 arcsec on a side, or about $5.7 \mathrm{kpc}$. $\mathrm{N}$ is up, $\mathrm{E}$ to the left. The image is reproduced in black and white in the printed version of the journal, but is available in colour in the electronic version.

region, but is very weak and only shows up in the $B-R$ colour index image (Fig. 4, lower left panel) and in the modelsubtracted $B$-band image of Fig. 9. To produce the latter, we subtracted an azimuthally constant model, based on the radial surface brightness profile, from the $B$ image. Both this and the colour index image show how rather chaotic spiral structure can be followed radially for at most another $1 \mathrm{kpc}$ into the outer disk. This spiral structure shows up in absorption: in red colour in the $B-R$ image, and as dark or less emitting regions in the model-subtracted $B$ image.

The spiral structure in the inner disk, or within $1.5 \mathrm{kpc}$ (27 arcsec) in radius, is prominent but rather flocculent (Figs. 5 and 10). The nuclear ring of radius $\sim 5$ arcsec identified by Pogge (1989) can be seen as an $\mathrm{H} \alpha$ ring in the FP moment zero maps (Figs. 2 and 11d). It seems to be slightly offset from the kinematic centre of the galaxy. This nuclear ring can also be recognised on the HST broad-band or colour index images, as regions with enhanced SF. Both the $\mathrm{H} \alpha$ total intensity map (Fig. 2) and the colour index images (Fig. 4) show that the SF activity within the inner disk area is biased toward the outer regions. This, coupled with the whispy spiral structure outlined in dust (red colours) in the colour index images, most spectacularly so in the HST image, leads to a similarity of NGC 278's inner region to nuclear ring regions in galaxies like M100 or NGC 5248 (e.g., Knapen et al. 1995a,b; Jogee et al. 2002a,b; see Buta \& Combes 1996 for a review on rings). We will discuss this nuclear ring in more detail in Sect. 4.5. 


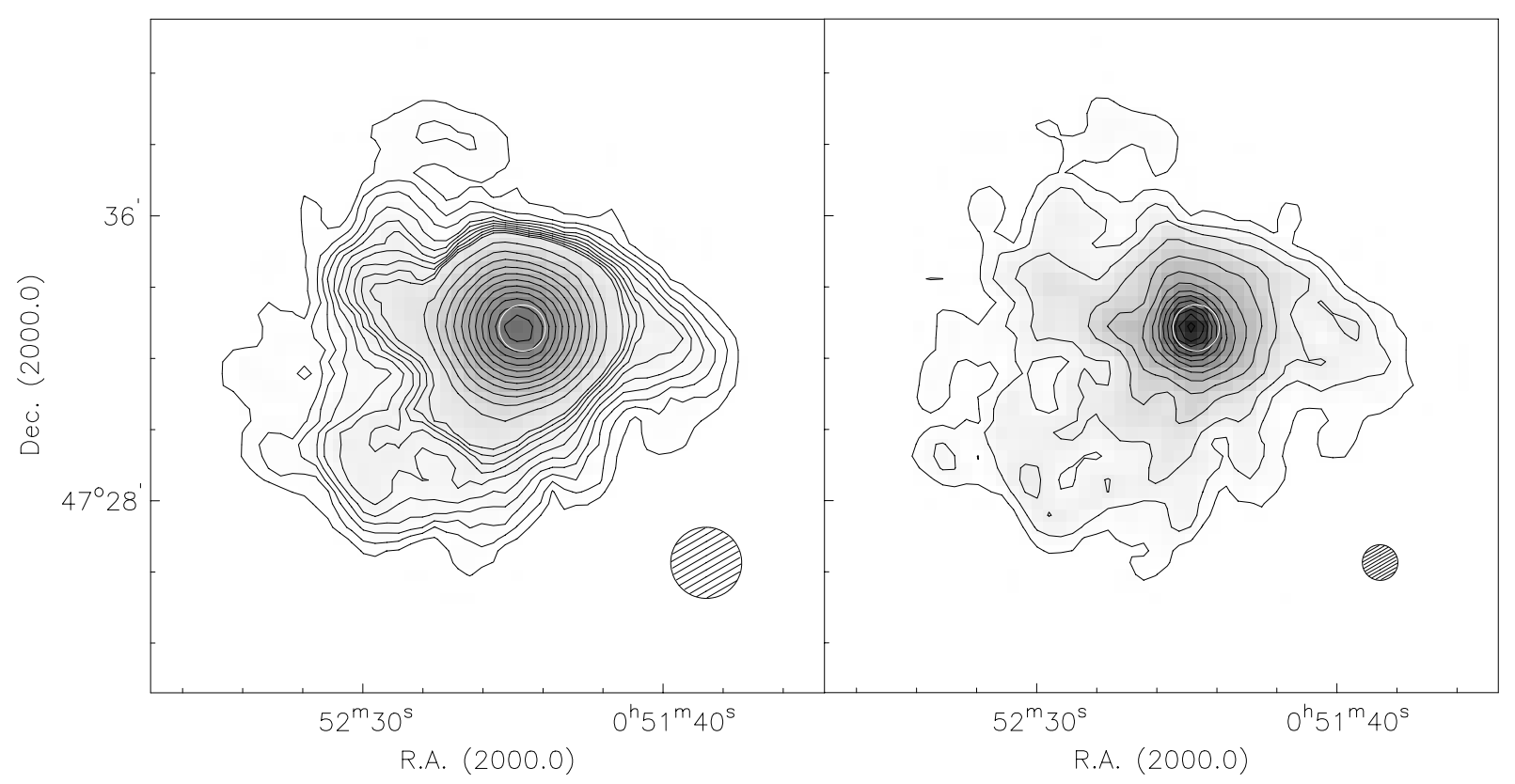

Fig. 6. Integrated $\mathrm{H}_{\mathrm{I}}$ surface density distributions at $120 \mathrm{arcsec}($ left $)$ and $60 \mathrm{arcsec}$ (right) resolution. In the left panel the contour levels are $(1,2,3, \ldots, 9) \times 10^{19},(1,1.5,2, \ldots, 6.5) \times 10^{20} \mathrm{~cm}^{-2}$. The contour levels in the right panel are $(0.2,0.5,1,2,3, \ldots, 11) \times 10^{20} \mathrm{~cm}^{-2}$. In both panels, the white contour indicates the extent of the optical component of NGC 278, as visible on the DSS. FWHM beam size is shown in the lower right corner of each panel.

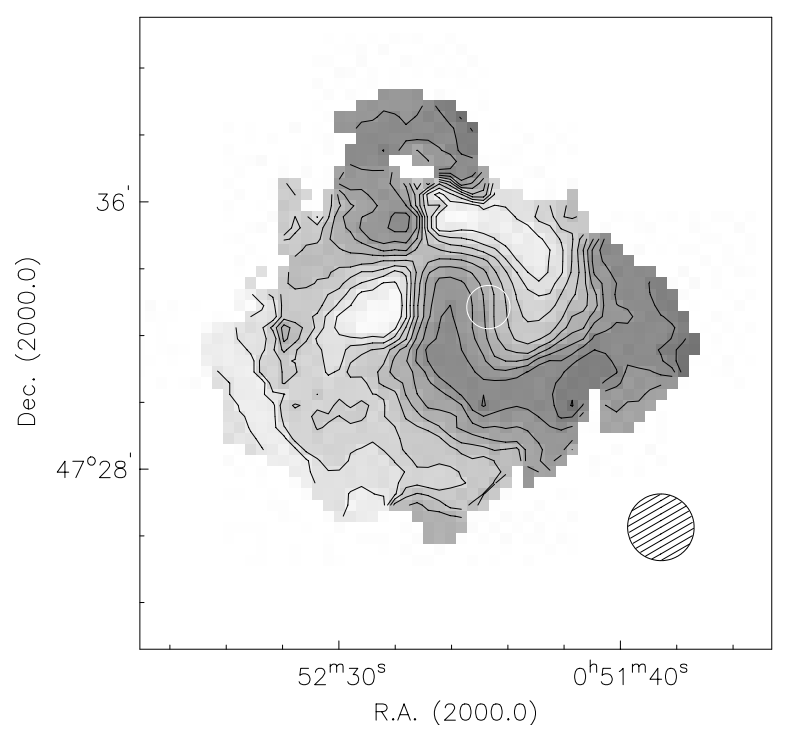

Fig. 7. Atomic hydrogen velocity field of NGC 278 at 120 arcsec resolution. Contour levels are from $620 \mathrm{~km} \mathrm{~s}^{-1}$ (light-grey) to $670 \mathrm{~km} \mathrm{~s}^{-1}$ (dark-grey) in steps of $5 \mathrm{~km} \mathrm{~s}^{-1}$.

\subsubsection{H I morphology of the outer regions}

The atomic hydrogen distribution of NGC 278 can be distinguished into two components: a compact (though in itself larger than the optical disk of the galaxy) central region of high column density, surrounded by an extended complex of H i clouds at low column density. The central part is readily visible at all resolutions, but the outer component is most clearly seen in the 120 arcsec and 60 arcsec resolution maps.

Figure 6 shows the integrated surface density maps of NGC 278 at 60 arcsec and 120 arcsec resolution. The column densities in the outer and inner parts differ by over a factor of 10 , with a sharp increase at the boundary between the two components. The extent of the optical component of NGC 278, as visible on a DSS image, is indicated in Fig. 6 as a white circle. The comparison illustrates clearly that the $\mathrm{H}_{\mathrm{I}}$ disk is very extended compared to the optical distribution. Due to the low spatial resolution needed to see the outer $\mathrm{H}$ I emission, it is hard to determine the detailed structure of the atomic hydrogen, but it seems from Fig. 6 that the $\mathrm{H}_{\mathrm{I}}$ is organised somewhat, possibly in arm fragments, of spiral or tidal origin.

These results are illustrated by Fig. 12, which shows the H I surface density profile of the 60 arcsec data, derived assuming an inclination of 17 degrees and a position angle (PA) of -50 degrees, which is the approximate PA of the kinematic major axis (at such low inclinations the precise value is not critical). The position where the $\mathrm{H}_{\mathrm{I}}$ distribution peaks was adopted as the centre.

The total flux of NGC 278 as derived from the 120 arcsec data is $31.7 \mathrm{Jy} \mathrm{km} \mathrm{s}^{-1}$, which agrees well with the single-dish value of $29.8 \mathrm{Jy} \mathrm{km} \mathrm{s}^{-1}$, as given by Huchtmeier \& Richter (1989). The corresponding $\mathrm{H}_{\mathrm{I}}$ mass is $1.1 \times 10^{9} M_{\odot}$. The mass of the bright central part (i.e., inside $180 \mathrm{arcsec}$ ) is $7.3 \times$ $10^{8} M_{\odot}$, that of the outer component $3.3 \times 10^{8} M_{\odot}$. The outer components thus contains some $30 \%$ of the total atomic hydrogen mass of the system.

\subsection{Kinematics}

\subsection{1. $\mathrm{H} \alpha$ kinematics in the inner region}

The velocity field of the inner disk region in NGC 278 (the only region available using $\mathrm{H} \alpha$ as a tracer, given the absence of $\mathrm{H} \alpha$ emission outside $r=27$ arcsec) is dominated by ordered 


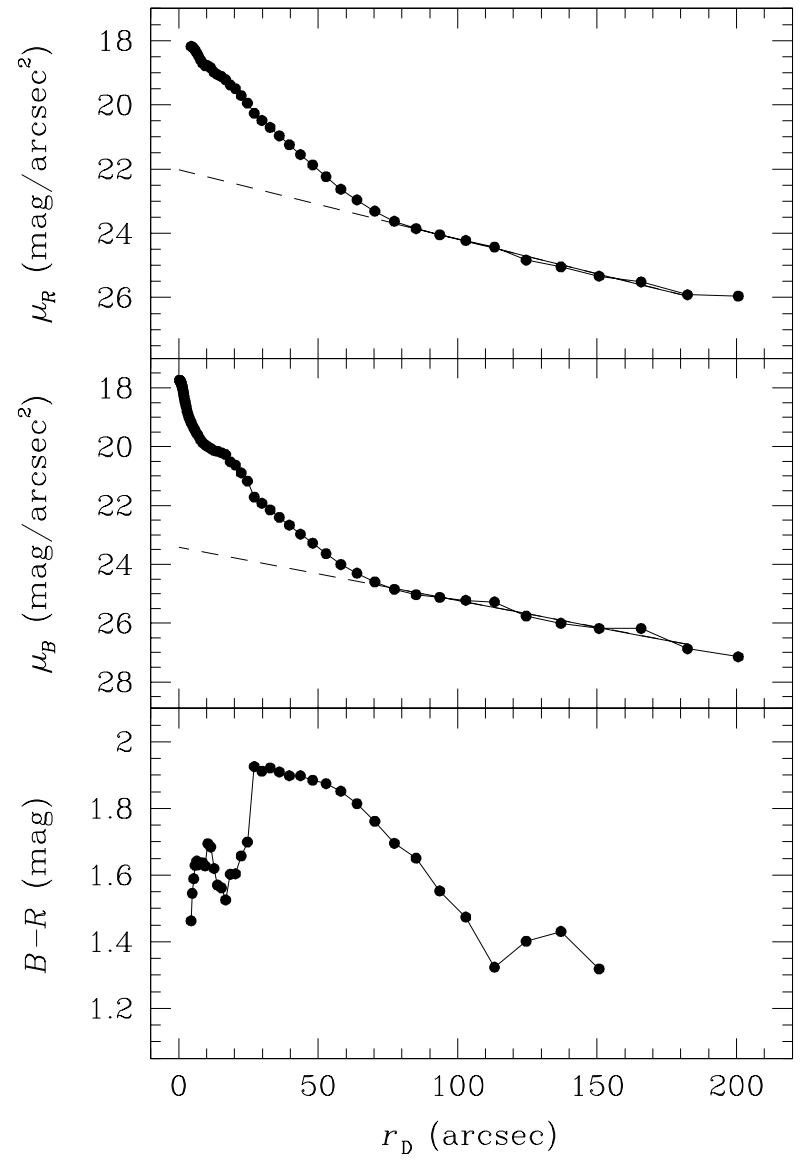

Fig. 8. Radial surface brightness profiles in the $R$ (top panel) and $B$ (middle panel) bands, and radial $B-R$ colour index profile (lower panel). Least-squares fits to the exponential scale lengths of the outer disk in $R$ and $B$ are indicated by the solid lines, $h_{R}=43 \operatorname{arcsec}, h_{B}=$ 51 arcsec, while the extrapolations of the fit inside the fitted range in radius are shown by dashed line.

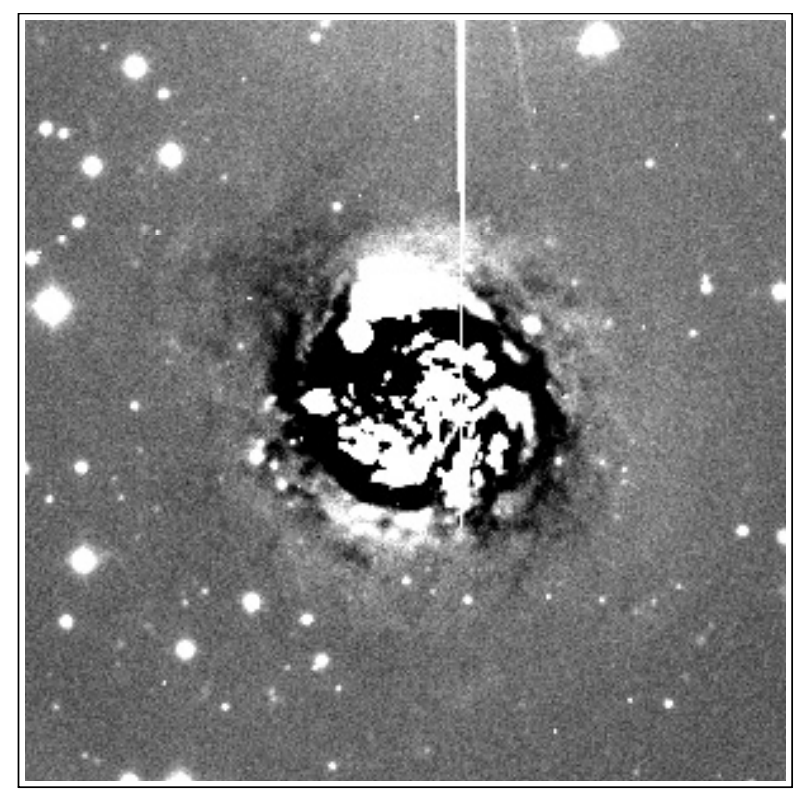

Fig. 9. $B$-band image of NGC 278 after subtracting an axisymmetric model derived from the radial surface brightness profile (Fig. 8). Area shown is 3 arcmin on the side.

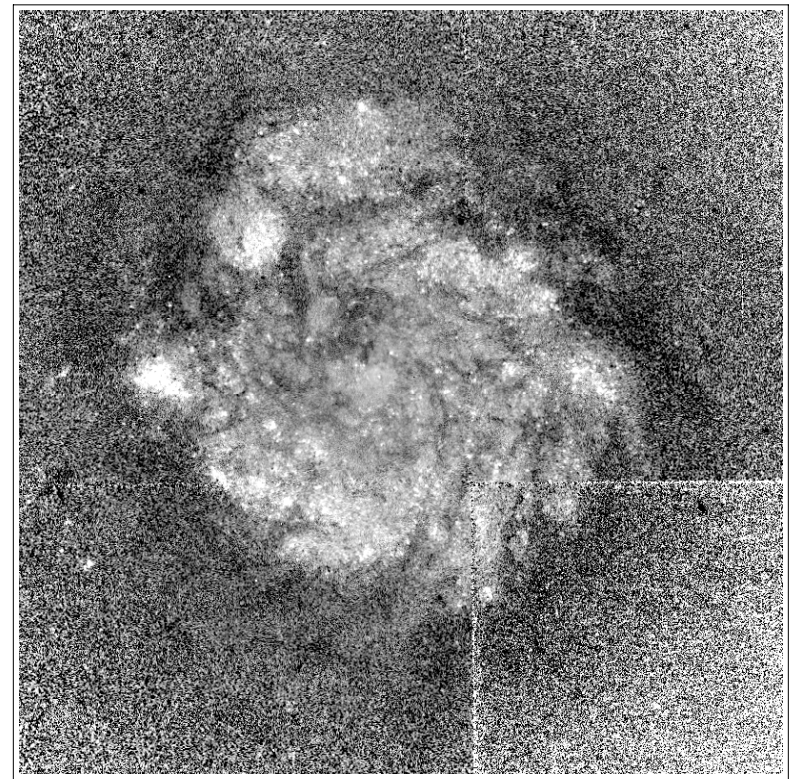

Fig. 10. $F 450 W-F 814 W H S T$ colour index image. Darker shades indicate redder colour.

circular motion (Figs. 2 and 11). Prominent non-circular, or rather non-regular, motions are seen, however, both in the form of the general clockwise rotation of the isovelocity contours toward the outer end of the area surveyed in $\mathrm{H} \alpha$, and in the form of small-scale deviations well within that area. The spatial coincidence of the small-scale deviations from circular motion with the spiral arms as traced by the integrated $\mathrm{H} \alpha$ emission (Figs. 2 and 11) suggests that these motions are induced by density wave streaming across the spiral arms. The PA of the kinematic minor axis in the central part of NGC 278 can be estimated from the velocity fields as 155 degrees. Closed isovelocity contours indicate that the circular rotation velocities reach a local maximum near a radius of 18 arcsec.

We derived rotation curves from the 1.2 and 3 arcsec resolution velocity fields, following the description of Knapen (1997). Because the galaxy is nearly face-on, the inclination angle cannot reasonably be constrained by the fitting procedure, and we assumed a constant inclination of 17 degrees. The resulting rotation curves are shown in Fig. 13 and show a relatively slow rise, and the local maximum of $\sim 120 \mathrm{~km} \mathrm{~s}^{-1}$ (or more accurately, 35/sin $i \mathrm{~km} \mathrm{~s}^{-1}$ ) at $r=18 \operatorname{arcsec}$ already seen from the velocity fields. The rotation curve at 3 arcsec resolution is also shown overplotted on the major axis positionvelocity plot, in Fig. 11e.

We used the latter curve, along with the fitted radial run of major axis PA (not shown), to produce a model velocity field, which can be assumed to trace circular motions. This model velocity field is shown, along with the original 3 arcsec resolution velocity field from which it was derived, in Fig. 11 (panels $b$ and a, respectively). Subtracting this model from the observed velocity field highlights the location and approximate amplitude of the residual, or non-circular, motions. This is shown in Fig. 11c, and overlaid on the total intensity $\mathrm{H} \alpha$ map in Fig. 11d. As described further by Knapen et al. (2000), the fact that the rotation curve used for producing the model velocity field 

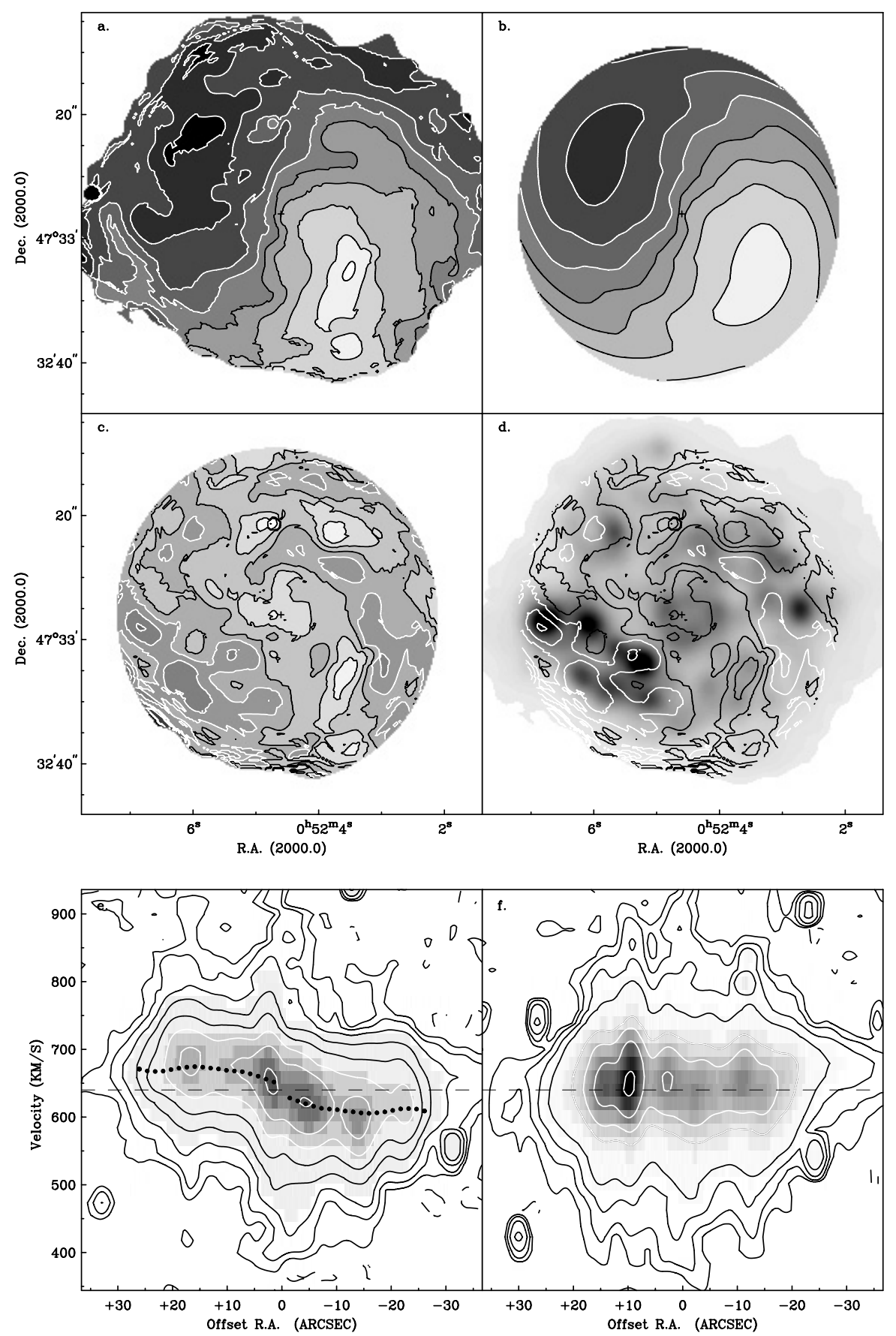

Fig. 11. a) (upper left) $\mathrm{H} \alpha$ FP velocity field of NGC 278 at 3 arcsec resolution. Grey-scale and contour levels are from 600 to $690 \mathrm{~km} \mathrm{~s}^{-1}$ in steps of $10 \mathrm{~km} \mathrm{~s}^{-1}$, with the lower velocity contours in black, and the first white contour at $650 \mathrm{~km} \mathrm{~s}^{-1}$. b) (upper right) Model velocity field as determined from the rotation curve (see text). Contour and grey levels as in Fig. 11a. c) (middle left) Residual velocity map, obtained by subtracting the model (Fig. 11b) from the velocity field (Fig. 11a). Contours are at $-5,0$ and $5 \mathrm{~km} \mathrm{~s}^{-1}$ (black) and 10,15 and $20 \mathrm{~km} \mathrm{~s}^{-1}$ (white), with grey-scales indicating the same range and higher values coded darker. d) (middle right) As Fig. 11c, now overlaid on a greyscale representation of the $\mathrm{H} \alpha$ total intensity, or moment zero, map at 3 arcsec resolution. e) (lower left) Position-velocity diagram along the kinematic majors axis of the central region ( $\mathrm{PA}=65$ degrees). Contour levels are at -4 and $-2 \sigma$ (dashed), 2, 4, 8, 16 and $32 \sigma$ (black), and 64,128 and $256 \sigma$ (white). Overlaid as black dots is the rotation curve for the entire disk at the same resolution. The systemic velocity of the galaxy is indicated by the horizontal dashed line. f) (lower right) As Fig. 11e, but now along the minor axis.

contains a certain contribution from the non-circular motions can lead to an underestimate of the non-circular motions in this technique. Here we will limit ourselves, however, to a qualitative discussion and this can be well based on the residual velocity field, as shown in Fig. 11.
This analysis shows that the clockwise skewing of the isovelocity contours near the outer end of the region observed in $\mathrm{H} \alpha$ can be reproduced with circular motions, albeit significantly changing the PA of the line of nodes. Because the effect occurs where the signal-to-noise ratio in the $\mathrm{H} \alpha$ data is 


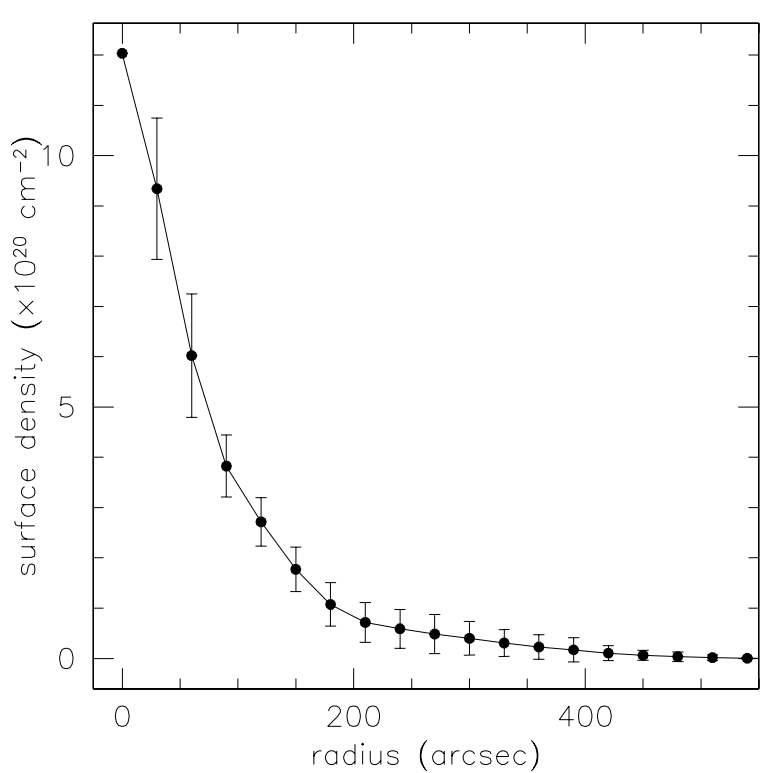

Fig. 12. H I surface density profile of NGC 278 at 60 arcsec resolution.

lowest, it must be treated with care, but in comparison to the non-regular Hi velocity field (Sect. 3.2.2) it is entirely reasonable to suspect that its origins lie in the same process that causes the $\mathrm{H}_{\mathrm{I}}$ velocity field to be so irregular, presumably a recent interaction as we will see below. Another possible explanation for the shape of the $\mathrm{H} \alpha$ velocity field could be the presence of a bar, but given that our imaging shows no evidence whatsoever of such a presence, this interpretation is unlikely.

Concentrating now on the regions of non-circular motion within the area mapped in $\mathrm{H} \alpha$, the residual velocity field shows, in white contours, that the most prominent of these regions occur to the SE, to the $\mathrm{W}$ and the ENE of the nucleus. Comparison with the $\mathrm{H} \alpha$, optical and NIR images, but especially with the HST image (Fig. 5) shows that this is where the most prominent spiral arm patches are located. This hints at an origin of the non-circular motion in density-wave induced streaming of gas across the spiral arms. The amplitude of the non-circular motions, of some $10-15 / \sin i \mathrm{~km} \mathrm{~s}^{-1}$, or $34-51 \mathrm{~km} \mathrm{~s}^{-1}$, is fully consistent with this interpretation, and although the spiral pattern may not at first sight look grand-design, colour index maps (Figs. 4 and 10), as well as the unsharp-masked image of Fig. 9, do show evidence for bi-symmetric spiral structure.

\subsubsection{H I kinematics in the outer disk}

We investigated the atomic hydrogen kinematics at all available resolutions, but found that the signal to noise ratio in the outer parts is too low to construct a meaningful velocity field in all but the lowest-resolution data sets. The H I velocity field of NGC 278 at 120 arcsec resolution is shown in Fig. 7. We stress that comparison with the high resolution ( 1 arcsec) velocity field in $\mathrm{H} \alpha$ is nearly impossible: the area mapped in $\mathrm{H} \alpha$, of at most 30 arcsec in radius, is in its entirety well within the area covered by the central beam in the $\mathrm{H}_{\mathrm{I}}$ data.

In the $\mathrm{HI}_{\mathrm{I}}$ velocity field, as in the $\mathrm{H}_{\mathrm{I}}$ column density maps, we can also distinguish two components: the inner part,

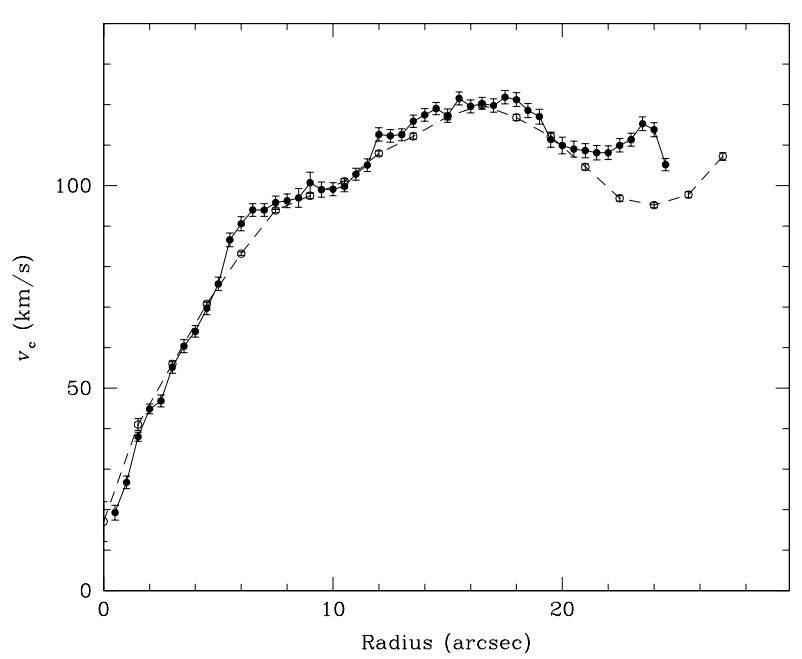

Fig. 13. Rotation curve as derived from the 1.2 arcsec (filled symbols and drawn line) and from the 3 arcsec (open symbols and dashed line) spatial resolution $\mathrm{H} \alpha$ velocity field of NGC 278.

corresponding to the high column density central region, shows a well-ordered velocity field, indicating a rotating disk, though with possibly a strong warp. Outside a radius of $\sim 3$ arcmin, the velocity field changes dramatically, and hardly any ordered rotation can be distinguished. The $\mathrm{H}_{\mathrm{I}}$ in these outer parts, however, does seem to form a coherent structure. Especially in the Eastern region, we see a continuity in velocity between the Northern and Southern parts, which suggests that this part of the $\mathrm{H}_{\mathrm{I}}$ distribution constitutes in NGC 278 something like a tidal stream. Unfortunately, the higher resolution data do not have enough $\mathrm{S} / \mathrm{N}$ to verify this idea in detail. There is also an intriguing continuity in velocity between the western part of the inner disk and the outer disk, perhaps suggestive of an infalling cloud. Given the absence of ordered motions, it is not meaningful to try and deduce a rotation curve, and by implication no information on the halo of this galaxy can be extracted from the velocity field. Some aspects of the H I velocity field are reminiscent of the effects a bar can have on an atomic hydrogen velocity field (e.g., Bosma 1980). As we will see below, however, it is much more likely that the deviations from circular motion, as well as the $\mathrm{H}_{\mathrm{I}}$ morphology and in fact the nuclear ring of SF, are a result of a past interaction.

Below, we will describe how the H I morphology and kinematics fit into our proposed scenario of a recent interaction as the origin of the currently observed structure of NGC 278.

\section{Discussion}

\subsection{Summary of observational results}

Before we discuss a plausible history of NGC 278, we briefly review the main observational findings. Together, these results outline the puzzle presented to us by this small galaxy.

1. NGC 278 possesses a large $\mathrm{H}_{\mathrm{I}}$ disk, with possibly in the outer regions the remains of a previously caught, gas-rich, companion.

2. In the central region, an area of active massive SF is found. 
3. Rather red colours in the optical disk may indicate an advanced age, and/or significant extinction.

4. The kinematics, both at small and large scales, are severely disturbed. In the outer disk, well outside the optical disk, the disturbance may be due to large-scale deviations from axisymmetry.

5. The disk has a low surface brightness, but its colour is much redder than the typical disk found in late-type low surface brightness galaxies (LSB galaxies).

6. The circumnuclear $\mathrm{H} \alpha$ morphology outlines a nuclear disk, which has a rather typical size in absolute terms, but is unusually large in comparison to the disk scale length or the size of its host galaxy.

In the following sections we will discuss the main features of this galaxy in view of the properties mentioned above. We will consider the surface brightness of the disk, the bright central part which shows little evidence for a bar, and the features directly related to the possibility of NGC 278 being the remnant of an accretion event: the large $\mathrm{H}_{\mathrm{I}}$ disk and the bright nuclear ring of SF.

\subsection{The low surface brightness disk of N278}

The disk of NGC 278 has a very low surface brightness: the extrapolated central value in the $B$-band $\left(\mu_{0, B}=\right.$ $23.5 \mathrm{mag} \operatorname{arcsec}^{-2}$ ) is comparable to that typically found for late-type LSB galaxies $\left(\mu_{0, B} \simeq 23.2 \mathrm{mag} \operatorname{arcsec}^{-2}\right.$; de Blok et al. 1995). As it has such a low surface brightness disk, a possible explanation for the current evolutionary state of NGC 278 could be that it is an LSB galaxy with much enhanced SF in the central few kiloparsec region, organised into spiral arms.

This is, however, not a likely scenario as the $B-R$ colour of the outer part of the optical disk of NGC 278 is about $1.5 \mathrm{mag}$ (see Fig. 8), redder than the average LSB galaxy which has a $B-R$ colour of 0.78 (de Blok et al. 1995). This is a significant difference given the small spread in $B-R$ exhibited by the LSB galaxies.

Comparing the LSB galaxies from de Blok et al. (1995) and the HSB galaxies from de Jong \& van der Kruit (1994) we find that NGC 278 is in a region of the $B-R$ vs. surface brightness diagram (e.g., Fig. 8 in de Blok et al. 1995 or Fig. 2.7 in de Blok 1995) which is only populated by a few faint galaxies from the de Jong \& van der Kruit sample. So the disk of NGC 278, despite its low surface brightness, must have a different SF history than the late-type LSB galaxies studied by de Blok et al. (1995).

\subsection{No bar}

The purpose of this short section is to reiterate that there is no evidence for the presence of a bar in NGC 278. Although the galaxy has been classified as "SAB" in the RC3, we see no evidence from our NIR (Fig. 3) or optical (Fig. 4) imaging. The NIR images only cover the innermost region of the disk, up to a radius of some 30 arcsec, and although some deviations from perfectly circular isophote shapes can be seen in the figure, these can, upon further inspection, be traced to localised regions of SF. We fitted ellipses to the NIR isophotes (not shown) and confirm from these what Fig. 3 shows qualitatively: there is no evidence for the presence of a bar in the area covered by the NIR images.

The optical images, shown in Fig. 4, cover a somewhat larger area of the disk, but also show absolutely no evidence for a bar from the isophotes (Fig. 4), nor indeed from ellipse fitting (not shown).

There are two other possibilities, both of which can be discarded as being rather implausible. The first is that a large bar is present, completely outside the range of our optical imaging, but which affects the velocity field of the outer part of the disk as seen in H I. Since such a bar would lie completely below a surface brightness level of $26 \mathrm{mag} \operatorname{arcsec}^{-2}$ in this nearby galaxy, it is not an attractive scenario, although we cannot formally rule it out. The second possibility is that there is a tiny nuclear bar which we cannot detect with our current imaging. HST NIR imaging is not available for NGC 278, but the WFPC2 optical imaging (Fig. 5) shows no indication whatsoever for the presence of a mini-bar. We conclude that there is no evidence for the presence of a bar in NGC 278.

\subsection{Possible merger history of NGC 278}

Following the prescription of Laine et al. (2002), we checked in the Lyon-Meudon extragalactic database (LEDA) whether NGC 278 has any nearby companions. Using a search radius of $400 \mathrm{kpc}$ around the galaxy, and imposing a $\pm 500 \mathrm{~km} \mathrm{~s}^{-1}$ range in $c z$, we found no companions. Extending our search to a wider area around NGC 278, we found the galaxy UGC 672, at $v_{\text {sys }}=708 \mathrm{~km} \mathrm{~s}^{-1}$, already discussed in the introduction (Sect. 1). This galaxy, however, is faint, with a magnitude of 18 given in NED, which would correspond to an absolute magnitude $M \sim-12.4$ mag. It is also at a distance of some $600 \mathrm{kpc}$ from NGC 278, and can thus not be considered a companion that could have had any effect on the evolution of NGC 278. NGC 278 is thus isolated, at least at present.

We have searched the $\mathrm{H}_{\mathrm{I}}$ data cubes for possible companions but did not detect any. This puts rather strong limits on the presence and masses of any possible companions, as well as on interaction timescales. Given that the noise in the 120 arcsec data is $7.45 \mathrm{mJy}$ beam $^{-1}$, and assuming that any undetected galaxies in the field have a $3 \sigma$ peak flux upper limit and a profile width of $50 \mathrm{~km} \mathrm{~s}^{-1}$, the flux of such an undetected galaxy would be $1.11 \mathrm{Jy} \mathrm{km} \mathrm{s}^{-1}$, which at the distance of NGC 278 corresponds to an upper limit in $\mathrm{H}_{\mathrm{I}}$ mass of $8.9 \times 10^{5} M_{\odot}$. The $3 \sigma$ column density limits in the 120 arcsec and 60 arcsec data are $7.1 \times 10^{18} \mathrm{~cm}^{-2}$ and $2.1 \times 10^{19} \mathrm{~cm}^{-2}$, respectively.

The Hi morphology and kinematics, however, especially in the outer parts of the disk, do yield evidence for a recent interaction. Both the asymmetrical morphology and disturbed kinematic structure of the disk are reminiscent of tidal tails and arms as found in numerical simulations of galaxy interactions (dating back to the classic paper by Toomre \& Toomre 1972).

Thus even though NGC 278 appears isolated at present, there is convincing evidence for a recent interaction. We speculate that this interaction may have been with a gas-rich dwarf, 
which has ceased to exist as such, and whose gaseous material now forms part of the tidal arm in the outer disk of NGC 278. The interaction may well have been of a similar nature to those studied by Hernquist \& Mihos (1995), who considered the case of a minor merger between a gas-rich disk and a dwarf galaxy, and who found that such a merger can lead to torquing and subsequent radial inflow of gas in the primary disk. The total atomic gas mass of NGC 278 at present, of $1.1 \times 10^{9} M_{\odot}$, would then be the total of the masses of the two individual galaxies before the collision, which implies that both must have been rather, but by no means implausibly, small galaxies. Assuming, as above, an absolute magnitude $M_{B}=-18.8$ implies that NGC 278 has an $\mathrm{HI}_{\mathrm{I}}$ mass to luminosity ratio $M_{\mathrm{HI}} / L_{B} \sim 0.4$. Such a ratio is about twice as high as expected for a typical Sb galaxy (Roberts \& Haynes 1994), and more typical of late-type Sd galaxies. This is an indication that significant gas infall has taken place at some time in the past. In view of the red colour of the disk, which indicates an advanced age, the event under consideration here has perhaps been the first merger event undergone by this galaxy.

\subsection{A nuclear ring in a non-barred galaxy}

Perhaps the most intriguing feature of this small spiral galaxy, NGC 278, is the contrast between the $2.2 \mathrm{kpc}$ diameter central region, with strong massive $S F$ activity and well defined spiral structure, and the region outside 27 arcsec in radius, where a mostly featureless disk without any noticeable SF extends to about $8 \mathrm{kpc}$ in radius in the optical, and much further as seen in $\mathrm{H}_{\mathrm{I}}$. The break between these two regions is caused by the sudden outward demise of SF and is abrupt, as seen in the images (Figs. 3-5) and in the radial profiles, especially of colour (Fig. 8). The spiral structure continues a bit into the outer disk, but in absorption (presumably caused by dust) more than in emission (Figs. 10 and 9). The $\mathrm{H} \alpha$ image (Fig. 2) and the radial colour profile, which suddenly becomes significantly redder (Fig. 8), constitute proof that the change in appearance between the inner and outer regions is indeed due to SF alone.

It is tempting to consider a star formation threshold defined by a Toomre criterion for the onset of star formation, following the analysis of Kennicutt $(1989,1998)$. But firstly, the drop in intensity, not just in the massive star formation tracer $\mathrm{H} \alpha$ but also in underlying optical light, is very large, larger than normally seen in spiral galaxies. Secondly, a Kennicutt-style analysis requires the observation of a rotation curve in order to calculate a critical gas density, and of a gas density profile to compare to the star formation profile. In NGC 278 , the velocity field is so different from well-ordered circular rotation that a rotation curve cannot be derived, whereas the edge of the star formation is well within the central beam of our neutral gas data.

As noted above, in Sect. 3.1, the appearance of the inner region, especially in colour index images and in the HST real-colour image (Figs. 3-5) is reminiscent of starforming nuclear rings in barred galaxies. Such nuclear rings are generally interpreted as dynamical effects of gas flow in the presence of ILRs which halt the net radial inflow of gas under the influence of a bar (e.g., Knapen et al. 1995a).

There are, however, a number of interesting differences between the nuclear ring in NGC 278 and those in other galaxies. First and foremost, the presence of nuclear rings is in most cases directly related to the presence of a bar (e.g. Buta \& Combes 1996), yet NGC 278 shows no trace of a bar. Second, the inner region in NGC 278 is relatively large for a nuclear ring. This is not so much the case for the diameter of the ring itself $(2.2 \mathrm{kpc})$, but rather in comparison to the size of the disk. The ring diameter is comparable to, or slightly larger than, the scale length of the exponential disk of the galaxy which hosts it, which is in stark contrast with the typical behaviour of nuclear rings, where the scale length is $3-10$ times larger than the ring diameter (e.g., Knapen et al. 2002). So whereas for NGC $278 h / D_{\text {ring }} \sim 1$, for instance, for NGC 4314 this ratio is 6.4 , for NGC 4321, 3.4, and for NGC 5248, 3.7 (Knapen et al. 2002). Another measure of ring size is its size relative to the size of the complete disk $\left(D_{25}\right)$. For NGC $278, D_{\text {ring }} / D_{25}$ is 0.31 , twice as large as the largest nuclear rings among the sample of 15 compiled by Knapen (2004). We thus find that the nuclear ring in NGC 278 is a relatively large nuclear ring, hosted by a non-barred and extremely small disk galaxy.

Buta \& Combes (1996) review the existence of rings (not only nuclear ones) in non-barred galaxies, and state that for most cases their host galaxies present evidence for some other kind of mechanism which sets up a non-axisymmetric potential, for instance a weak oval distortion, or the tidal action of a bound companion. We believe that this is also the case here, where our favoured explanation for the origin of the nuclear ring in NGC 278 is a non-axisymmetric potential, set up as a result of the minor merger with a companion. This potential then has a very similar effect to one set up by a classical bar, and can result in a nuclear ring. NGC 278 thus represents a case for the effects of interactions on the gas distribution within galaxies, and for the formation of a nuclear ring in a non-barred galaxy.

Finally, NGC 278 hosts a small ring inside the inner region, as seen in $\mathrm{H} \alpha$ (Sect. 3.1), at a radius of $0.2 \mathrm{kpc}$. We have found a very similar case in the galaxy NGC 5248. There, a large bar hosts a set of two rings as seen in $\mathrm{H} \alpha$ (Laine et al. 2001; Maoz et al. 2001), at radii of 0.45 and $0.1 \mathrm{kpc}$ (Jogee et al. 2002b) (where the scale length of the exponential disk is $3.3 \mathrm{kpc}$; Knapen et al. 2002). Such mini-rings may be more common than is now recognised because they can only be found from high resolution imaging of nearby galaxies, preferably in emission lines which trace massive SF. Possible origins for such mini-rings can lie in the dynamical structure set up by the host galaxy, similar to classic nuclear rings forming between a pair of ILRs, or in SF triggered by outflow resulting from nuclear starburst or even non-stellar activity.

\section{Conclusions}

Using a combination of FP $\mathrm{H} \alpha$ data, optical and NIR imaging, and atomic hydrogen mapping, we make a detailed study of the small spiral galaxy NGC 278. The main conclusions from our work can be summarised as follows. 
1. NGC 278 is a small face-on spiral galaxy, of optical diameter only $7.2 \mathrm{kpc}$. Most of this disk is not actively forming stars, and the SF is concentrated within the central $1.5 \mathrm{kpc}$ radius.

2. The massive circumnuclear SF is organised in a nuclear ring, which contains a set of patchy spiral arms. The $\mathrm{H} \alpha$ velocity field shows some streaming motions due to these spiral arms.

3. The nuclear ring in NGC 278 is not particularly large in absolute terms (radius of one kpc) but is at least twice as large as other nuclear rings when its size relative to that of the host galaxy is considered, or its size relative to the scale length of the disk of its host galaxy.

4. We find no evidence for the presence of a bar in NGC 278, at any scale.

5. At present, NGC 278 does not have any companions which can gravitationally affect it.

6. The H I velocity field shows large deviations from circular motion. We also observe an irregular structure of the atomic hydrogen morphology, and interpret these findings as evidence for a recent merger with a small gas-rich galaxy.

7. We postulate that this interactive event has led to a nonaxisymmetry in the gravitational potential, which in turn has facilitated the formation of the nuclear ring. This explains why a nuclear ring can exist in a non-barred galaxy.

Acknowledgements. We thank Seppo Laine for his help with the reduction of the FP data, Jim Lewis for help with the TAUCAL reduction software, and Dolores Pérez-Ramírez, René Doyon and Daniel Nadeau for their help with the observations. Sharon Stedman kindly fitted the radial profiles to the INT data, and Nik Szymanek produced the real-colour image of NGC 278 (Fig. 5). We thank Isaac Shlosman and Elias Brinks for comments on an earlier version of this manuscript. L.F.W. thanks the Department of Physical Sciences of the University of Hertfordshire (UH) and the ING for financial help for a visit to the ING where this work was advanced. The present paper includes results from a final year project by L.F.W. at UH, for which support from UH is acknowledged. The William Herschel and Isaac Newton Telescopes are operated on the island of La Palma by the ING in the Spanish Observatorio del Roque de los Muchachos of the Instituto de Astrofísica de Canarias. The WHISP project has been supported by the Kapteyn Institute and the Foundation for Research in Astronomy (ASTRON) and has been carried out with the WSRT. The WSRT is operated by ASTRON with financial support from the Netherlands Organisation for Scientific Research (NWO). We used data obtained with the Canada-France-Hawaii Telescope, operated by the National Research Council of Canada, the Centre National de la Recherche Scientifique de France and the University of Hawaii. Partly based on observations made with the NASA/ESA Hubble Space Telescope, obtained from the data archive at the Space Telescope Science Institute, which is operated by the Association of Universities for Research in Astronomy, Inc., under NASA contract NAS 5-26555.

\section{References}

Arp, H., \& Sulentic, J. W. 1985, ApJ, 291, 88

Bosma, A. 1981, AJ, 86, 1825

Bushouse, H. A., Werner, M. W., \& Lamb, S. A. 1988, ApJ, 335, 74

Buta, R., \& Combes, F. 1996, Fund. Cosmic Phys., 17, 95

de Blok, W. J. G. 1995, Ph.D. Thesis, University of Groningen de Blok, W. J. G., van der Hulst, J. M., \& Bothun, G. D. 1995, MNRAS, 274, 235

de Vaucouleurs, G., de Vaucouleurs, A., Corwin, H. G., et al. 1991, Third reference catalogue of bright galaxies (New York: Springer) (RC3)

Frogel, J. A., Persson, S. E., Matthews, K., \& Aaronson, M. 1978, ApJ, 220, 75

Garrido, O., Marcelin, M., Amram, P., \& Boissin, O. 2003, A\&A, 399, 51

Heller, C. H., \& Shlosman, I. 1996, ApJ, 471, 143

Hernquist, L., \& Mihos, J. C. 1995, ApJ, 448, 41

Huchtmeier, W. K., \& Richter, O.-G. 1989, A general catalog of HI observations of galaxies. The reference catalog (Berlin, Heidelberg, New York: Springer-Verlag)

Jogee, S., Knapen, J. H., Laine, S., et al. 2002a, ApJ, 570, L55

Jogee, S., Shlosman, I., Laine, S., et al. 2002b, ApJ, 575, 156

de Jong, R. S., \& van der Kruit, P. C. 1994, A\&AS, 106, 451

Karachentseva, V. E., Prugniel, P., Vennick, J., et al. 1996, A\&AS, 117,343

Kennicutt, R. C. 1989, ApJ, 344, 685

Kennicutt, R. C. 1998, ApJ, 498, 541

Knapen, J. H. 1997, MNRAS, 286, 403

Knapen, J. H. 2004, A\&A, submitted

Knapen, J. H., Beckman, J. E., Heller, C. H., Shlosman, I., \& de Jong, R. S. 1995a, ApJ, 454, 623

Knapen, J. H., Beckman, J. E., Shlosman, I., et al. 1995b, ApJ, 443, L73

Knapen, J. H., Shlosman, I., Heller, C. H., et al. 2000, ApJ, 528, 219

Knapen, J. H., Pérez-Ramírez, D., \& Laine, S. 2002, MNRAS, 337, 808

Laine, S., Knapen, J. H., Pérez-Ramírez, D., Englmaier, P., \& Matthias, M. 2001, MNRAS, 324, 891

Laine, S., Shlosman, I., Knapen, J. H., \& Peletier, R. F. 2002, ApJ, 567, 97

Maoz, D., Barth, A. J., Ho, L. C., Sternberg, A., \& Filippenko, A. V. 2001, AJ, 121, 3048

Nadeau, D., Murphy, D. C., Doyon, R., \& Rowlands, N. 1994, PASP, 106, 909

Pease, F. G. 1917, ApJ, 46, 24

Pérez-Ramírez, D., Knapen, J. H., Peletier, R. F., et al. 2000, MNRAS, 317,234

Phillips, A. C., Illingworth, G. D., MacKenty, J. W., \& Franx, M. 1996, AJ, 111, 1566

Pogge, R. W. 1989, ApJS, 71, 433

Roberts, M. S., \& Haynes, M. P. 1994, ARA\&A, 32, 115

Schmidt, A. A., Bica, E., \& Alloin, D. 1990, MNRAS, 243, 620

Shlosman, I. 1999, in The evolution of galaxies on cosmological timescales, ed. J. E. Beckman, \& T. J. Mahoney (San Francisco: ASP), ASP Conf. Ser., 187, 100

Toomre, A., \& Toomre, J. 1972, ApJ, 178, 623

Tully, R. B. 1988, Nearby galaxies catalog (Cambridge and New York: Cambridge University Press)

Vallee, J. P. 1993, MNRAS, 264, 665

van der Hulst, J. M. 2002, in Seeing through the dust: The detection of $\mathrm{H}_{\mathrm{I}}$ and the exploration of the ISM in galaxies, ed. A. R. Taylor, T. L. Landecker, \& A. G. Willis (San Francisco: ASP), ASP Conf. Ser., 276, 84

van der Hulst, J. M., van Albada, T. S., \& Sancisi, R. 2001, in Gas and Galaxy evolution, ed. J. E. Hibbard, M. Rupen, \& J. H. van Gorkom (San Francisco: ASP), ASP Conf. Ser., 240, 451 